\title{
Liver disease in cystic fibrosis
}

\author{
Natalia Kobelska-Dubiel, Beata Klincewicz, Wojciech Cichy \\ Department of Paediatric Gastroenterology and Metabolic Diseases, Poznan University of Medical Sciences, Poland
}

Prz Gastroenterol 2014; 9 (3): 136-141

DOI: $10.5114 /$ pg.2014.43574

Key words: cystic fibrosis, liver disease, diagnostics, treatment.

\begin{abstract}
Address for correspondence: Natalia Kobelska-Dubiel, Department of Paediatric Gastroenterology and Metabolic Diseases, Poznan University of Medical Sciences, 27/33 Szpitalna St, 60-572 Poznan, Poland, phone: +48 618491 432, fax: +48 618472685 , e-mail: natalia.kobelska@gmail.com
\end{abstract}

\begin{abstract}
Cystic fibrosis-associated liver disease (CFLD) affects ca. 30\% of patients. The CFLD is now considered the third cause of death, after lung disease and transplantation complications, in CF patients. Diagnostics, clinical assessment and treatment of CFLD have become a real challenge since a striking increase of life expectancy in CF patients has recently been observed. There is no elaborated "gold standard" in the diagnostic process of CFLD; clinical evaluation, laboratory tests, ultrasonography and liver biopsy are used. Clinical forms of CFLD are elevation of serum liver enzymes, hepatic steatosis, focal biliary cirrhosis, multilobular biliary cirrhosis, neonatal cholestasis, cholelithiasis, cholecystitis and micro-gallbladder. In children, CFLD symptoms mostly occur in puberty. Clinical symptoms appear late, when damage of the hepatobiliary system is already advanced. The CFLD is more common in patients with severe mutations of CFTR gene, in whom a complete loss of CFTR protein function is observed. CFLD, together with exocrine pancreatic insufficiency and meconium ileus, is considered a component of the severe CF phenotype. Treatment of CFLD should be complex and conducted by a multispecialist team (gastroenterologist, hepatologist, dietician, radiologist, surgeon). The main aim of the treatment is to prevent liver damage and complications associated with portal hypertension and liver cirrhosis. Ursodeoxycholic acid is used in the treatment of CFLD. There is no treatment of proven long-term efficacy in CFLD. Liver transplantation is a treatment of choice in end-stage liver disease.
\end{abstract}

\section{Introduction}

Cystic fibrosis (CF) is the most common genetic disease occurring in Caucasians, of autosomal-recessive inheritance. It is a systemic disease, in the course of which pathologies of different systems and organs can be observed. The severity of the disease, and the survival time of patients, is determined by the degree of changes in the respiratory system. In recent years, due to progress in the treatment of cystic fibrosis, the lifespan of such patients has been prolonged. This can lead to a display of pathologies of other systems and organs that were not previously observed in this disease due to the short duration of the patients' life.

Cystic fibrosis-associated liver disease (CFLD) belongs to the group of common symptoms of this disease; however, due to the lack of specific and sensitive CFLD diagnostic markers, the epidemiological data may be incomplete. According to various sources, the prevalence rate of CFLD, diagnosed on the basis of clinical, biochemical and imaging (ultrasonography) tests, is $2-37 \%$ in children and young adults [1]. Currently it is believed that CFLD is the third most frequent cause of death in patients with cystic fibrosis, after lung disease and complications related to organ transplantation. This percentage, according to different sources, is about 2-4\% [1].

\section{Pathomechanism of cystic fibrosis-associated liver disease}

The pathomechanism of changes observed in the liver and in the bile ducts in patients with cystic fibrosis has yet to be completely understood. CFTR gene expression in the hepatobiliary system occurs only at the level of the epithelium of the bile ducts and gallbladder, 
while it does not occur in the hepatocytes and other cells of the liver. However, pathological lesions in the liver and the bile ducts in the course of CFLD remain in a close cause-and-effect relationship. The CFTR protein is anchored in the apical membrane of the cholangiocyte, regulating the content of water and electrolytes in the bile by affecting the transport of sodium and chloride ions and the secretion and alkalinisation of bile. Bile alkalinisation has a decisive influence on digestive functions and the solubility of its organic components. An abnormal CFTR protein in the biliary system may lead to the impaired secretion and deposition of thick, viscous bile with reduced alkalinity. Abnormal bile features the increased activity of free radicals, and its stagnation increases the susceptibility to infectious agents and other toxic components secreted with the bile. The accumulation of hydrophilic, toxic bile acids may damage a hepatocyte directly. In addition, during the course of CFLD, disorders of mucin secretions occur (leading to increased secretion of chondroitin sulphate) followed by obstruction of the small bile ducts [2]. An abnormal composition of the bile and its reduced flow leads to damage of cholangiocytes and periductal inflammation, bile duct proliferation and periportal fibrosis. Hepatic stellate cells (the main "culprits" of hepatic fibrosis) are activated and start to produce collagen, as well as stimulating the epithelium of bile ducts to release cytokines, which intensify the fibrosis processes, such as transforming growth factor- $\beta$ (TGF- $\beta$ ) [3]. This process, over a number (or even tens) of years, may lead to the development of initially focal and then multilobular cirrhosis with accompanying portal hypertension. It is believed that this problem concerns about $2-8 \%$ of patients with cystic fibrosis [3].

Minagawa et al. [4] have suggested that the CFTR protein affects the release of ATP to the lumen of the bile ducts, which in turn regulates the secretion of cholangiocytes through activation of the purinergic receptors (P2Y). In turn, Fiorotto et al. [5] demonstrated that the choleretic effect of ursodeoxycholic acid (UDCA) is related to CFTR-dependent ATP secretion. It is believed that the reduced flow of bile resulting from the CFTR protein dysfunction may also be linked to disorders affecting other mechanosensitive channels, intensifying disorders of chloride ion secretion and bile production [3].

\section{Genetic factors in cystic fibrosis-associated liver disease}

So far, more than 2000 mutations of the CFTR gene have been discovered, and these can be divided into five classes based on their impact on the CFTR protein function. The most common mutation, occurring in about $60-70 \%$ of patients with CF, is F508del, which dominates in Europe (including Poland) and North America. This mutation belongs to class II, and is associated with the deletion of three nucleotides - CTT (cytosine-thymine-thymine), which leads to the deletion of phenylalanine in position 508 in the protein product [6]. It is believed that abnormalities of the hepatobiliary system occur almost solely in patients with severe mutations, belonging to classes I-III, which leads to the impaired synthesis, modification or regulation of the CFTR protein [7]. For this reason, recently it has been emphasised that CFLD, along with exocrine pancreatic insufficiency and meconium ileus, is a constituent of severe cystic fibrosis phenotype [8]. However, the genotype-phenotype correlation that predicts the mutation effects on the clinical expression of CFLD is not possible at this stage. Therefore, it is suggested that genetic testing should not be used as a screening test for diseases of the hepatobiliary system in patients with cystic fibrosis [3]. It still remains unclear why only in a small number of patients with the same severe mutations symptoms of CFLD develop. It is believed that this is influenced by a number of other factors, such as age at the time of diagnosis, male sex, history of meconium ileus or exocrine pancreatic insufficiency [9]. A history of meconium ileus increases the risk of CFLD development by up to five times, with early symptoms being observed at the average age of 5 years, while in patients without this complication the average age was 8 years [8].

\section{Pathogenic factors in cystic fibrosis-associated liver disease}

Colombo et al. [8] observed CFLD symptoms in a group of patients, appearing in all girls before the period of puberty and suggesting the probable impact of endocrine factors (including oestrogens) on the process of proliferation of bile duct epithelium in response to liver damage. Estrogens and their receptors play a key role in the proliferation and secretion processes of the intrahepatic biliary epithelium. Some authors suggest that the impact on CFLD occurrence in patients with cystic fibrosis may include extensive surgery procedures and total parenteral nutrition $[10,11]$. In the pathogenesis of CFLD the existence of a correlation in the degree of pancreatic sufficiency has been suggested: CFLD is generally not observed in patients with pancreatic sufficiency. An impact of other genetic and environmental factors on the expression and clinical course of CFLD has been considered. It was suggested that histocompatibility complex antigens, infective agents, as well 
Table I. Clinical picture of CFLD (CF-associated liver disease) [2]

\begin{tabular}{|c|c|}
\hline Clinical type & $\begin{array}{c}\text { Estimated } \\
\text { frequency (\%) }\end{array}$ \\
\hline \multicolumn{2}{|l|}{ Liver: } \\
\hline $\begin{array}{l}\text { Asymptomatic elevation of liver } \\
\text { enzymes in serum }\end{array}$ & $10-35$ \\
\hline Hepatic steatosis & $20-60$ \\
\hline Focal biliary cirrhosis & $11-70$ \\
\hline Multilobular biliary cirrhosis & $5-15$ \\
\hline Neonatal cholestasis & Rare \\
\hline \multicolumn{2}{|l|}{ Gallbladder: } \\
\hline Cholelithiasis and cholecystitis & $1-10$ \\
\hline Micro-gallbladder & 30 \\
\hline
\end{tabular}

as a use of different therapeutic methods, might play a role in this process [2].

\section{Clinical presentation of cystic fibrosis-associated liver disease}

Epidemiological studies show that the clinically significant pathology of the hepatobiliary system in patients with cystic fibrosis usually develops before or during adolescence, its incidence is 15-17\% (although isolated hepatomegaly may be observed in $6-30 \%$ of cases), and is it characterised by a slow but progressive clinical course [12]. The clinical picture of CFLD can take many forms, from mild asymptomatic hypertransaminasaemia to cirrhosis (Table I).

In infants with cystic fibrosis, liver dysfunction may occur in the form of cholestasis. Cholestasis in infants occurs relatively rarely; in about $50 \%$ of cases it co-occurs with meconium ileus and parenteral nutrition. In the pathomechanism of this phenomenon, the thickening processes of acidophilic secretion in the intrahepatic bile ducts are taken into consideration. In laboratory tests, an increased concentration of conjugated bilirubin clinically accompanied by discoloured stools may be observed, which may incorrectly suggest a diagnosis of atresia of the extrahepatic bile ducts. The symptoms usually relieve spontaneously; however, in some patients liver fibrosis may develop [2].

In patients with cystic fibrosis, other irregularities within bile ducts are also observed, such as cholelithiasis and cholecystitis, the incidence of which increases with age. There is often a reduction in the gallbladder dimension, which, together with increased bile viscosity, promotes the development of cholelithiasis. The small gallbladder is mostly asymptomatic and is only incidentally detected in ultrasound tests. In some adult patients with cystic fibrosis, symptoms similar to those of primary sclerosing cholangitis (PSC) occur. It is believed that the frequency of CFTR gene mutation in the population of patients with PSC is $10.6 \%$, which is higher than in the general population at $4 \%$. In some patients, common bile duct obstruction may also occur, manifesting as abdominal pain and jaundice [2].

In about $10-30 \%$ of patients with CF in the initial period of puberty, asymptomatic hepatomegaly occurs, the most commonly connected with fibrosis processes of this organ. Bleeding from the upper part of the digestive tract rarely occurs as a first symptom of CFLD, which is connected with a focus on early identification of CFLD risk factors, particularly portal hypertension [2].

In older children and adults, CFLD includes three stages of advanced pathological lesions: hepatic steatosis accompanied (or not) by hepatitis, focal biliary cirrhosis and multilobular cirrhosis of the liver.

Among many pathologies of the hepatobiliary system that may occur in the course of CF, hepatic steatosis is one of the most common, occurring in the range 23$75 \%$ of cases [9]. The causes of hepatic steatosis secondary to cystic fibrosis have yet to be fully explored. Pathogenesis is not directly connected with a defective CFTR gene, but it may be related to malnutrition, deficiencies of essential fatty acids, carnitine or choline, oxidative stress as well as insulin resistance, commonly occurring in patients with cystic fibrosis [3]. It is not fully clear whether hepatic steatosis is progressive and inevitably leads to liver cirrhosis, or whether this process is reversible. Clinically, hepatic steatosis manifests as liver enlargement, and ultrasound examination exposes the hyperechogenic structure of the liver tissue. Biochemical tests confirm the increased activity of transaminases (ALT, AST). It is worth emphasising that normal size of the liver and correct transaminase activity is not unequivocal in excluding hepatic steatosis. The treatment of hepatic steatosis should be aimed at improving the nutritional status of the patient, including supplementation with fat-soluble vitamins, particularly in patients with associated cholestasis. Due to the fact that hepatic steatosis can be asymptomatic during the initial stage, ultrasound examination of the abdominal cavity should be performed at least once a year in all patients with cystic fibrosis, as well as a basic biochemical evaluation of liver function.

Cirrhosis is a final, irreversible stage of liver damage that leads to the failure of the organ. The clinical presentation of advanced liver cirrhosis includes jaundice, coagulation process disorders, ascites and the development of portal hypertension. Children with cystic fibrosis develop portal hypertension and oesophageal varices in the course of cirrhosis, linked to a risk of haemorrhage that 
may cause an immediate and vital risk. Portal hypertension is accompanied by splenomegaly with a decreased number of thrombocytes and leukocytes. The activity of hepatocytes is maintained, symptoms of liver failure are not observed and in laboratory tests, except for the above-mentioned haematological irregularities (thrombocytopaenia, leukopaenia), aberrations are often not observed. Biochemical exponents of liver failure (increased level of bilirubin, decreased concentration of albumins and extension of prothrombin time) appear late in advanced liver failure. Ultrasonography is a primary test carried out in patients with suspected cirrhosis of the liver.

\section{Cystic fibrosis-associated liver disease diagnostics}

The CFLD diagnosis may cause many difficulties as a "gold standard test" has yet to be developed. Early diagnosis of CFLD is very important because clinical symptoms appear late, when hepatobiliary system damage is already very advanced. Some studies suggest that only during the early stages are histopathological changes reversible and may be efficaciously treated. In everyday clinical practice, four tools for identification of CFLD are commonly used: (1) regular clinical evaluation, (2) evaluation of liver enzyme activity, (3) measurement of prothrombin time and (4) ultrasonography. In some centres, to assess the advancement of the disease, an endoscopic test of upper gastrointestinal tract (oesophagus varices and stomach evaluation) is performed, as well as a histological test of liver bioptates [2, 13].

Colombo et al. [according to 3] developed diagnostic criteria for CFLD. For these criteria, CFLD diagnosis should be based on a positive result from the CFLD histological test (characteristics of focal and multilobular biliary cirrhosis) or should meet at least two of the following criteria, evaluated at least twice a year: (1) the presence of hepatomegaly ( $>2 \mathrm{~cm}$ below the costal margin in the midclavicular line) confirmed by ultrasound test; (2) abnormal results of liver enzyme activity obtained twice (ALT, AST or GGTP); and (3) confirmed abnormalities other than hepatomegaly in the ultrasound test (increased, heterogenic echogenicity of tissue, tuberositas, irregular edges and splenomegaly) [3].

Comprehensive clinical evaluation of a patient with cystic fibrosis, observed for CFLD, should be focused on the occurrence of the following symptoms: problems with gaining weight (children), fatigue, nausea, abdominal distension, abdominal pain, purpura, gastrointestinal track bleeding, jaundice and pruritus. The physical examination should include: palpation of the liver with an assessment of its size and cohesiveness, percussion, evaluation of the spleen size, observation of skin lesions in the course of chronic liver disease (palmar erythema, spider veins, jaundice, clubbed fingers and extended abdominal subcutaneous veins), evaluation of ascites, the presence of neurological symptoms of cirrhosis and evaluation of nutritional status [2].

Biochemical tests are also used to establish the diagnosis of CFLD. In patients with CFLD, laboratory exponents of cholestasis (GGTP, ALP) are more commonly observed than the abnormal activity of transaminases. It is believed that hypertransaminasaemia is not a sufficiently sensitive and specific exponent of CFLD, as it can show mild severity or occur temporarily, and correlates poorly with the degree of severity of liver damage. About $20-30 \%$ of children tested in any period may have an isolated increase in transaminase activity. Other, less frequently used biochemical tests used in the evaluation of CFLD are: aminopyrine breath test, capacity to eliminate galactose test, caffeine tolerance test, measurement of serous glutathione S-transferase $\mathrm{BI}$ activity, concentration of collagen $\mathrm{V}$ and prolyl hydroxylase activity (markers of fibrogenesis) [2].

Among the imaging tests in patients with clinical symptoms or laboratory exponents of CFLD, ultrasonography plays an important role in the diagnostic process. This test allows the evaluation of focal biliary fibrosis, multilobular cirrhosis, hepatic steatosis, biliary duct abnormalities and abnormalities of blood flow in the portal vein (Doppler US). In some patients, a liver biopsy is helpful in the diagnosis of CFLD. The CFLD pathognomonic lesions in the hepatic tissue are focal biliary cirrhosis. A liver biopsy allows one to differentiate between focal biliary cirrhosis and hepatic steatosis; it allows the assessment of the extension of fibrosis processes and allows the exclusion of other causes of liver dysfunction. Some researchers believe that, due to the focal nature of the hepatic tissue lesions in the course of CFLD, a percutaneous biopsy of this organ cannot be considered as a "gold standard" and, additionally, that there is some risk $(0.03 \%$ of mortality and 3\% of morbidity). Contraindications to performing this procedure are thrombocytopaenia and ascites (in these situations either a laparoscopic approach or via the cervical vein may be considered) [2].

Other imaging tests used in the diagnosis and assessment of CFLD advancement include: scintigraphy (evaluates abnormal biliary drainage, progression of the disease and response to treatment), endoscopic retrograde cholangiopancreatography - ERCP (invasive test with a risk of complications, useful in evaluating bile duct abnormalities), and magnetic resonance cholangiopancreatography - MRCP (useful when ERCP does not allow full assessment of the pathology) [2].

Currently it is believed that CFLD should be treated as an early complication of cystic fibrosis. For this 
Table II. Yearly laboratory tests recommended to identify CFLD [2]

\begin{tabular}{l} 
Complete blood count \\
\hline Prothrombin time (PT) \\
\hline Aspartate aminotransferase (AST) \\
\hline Alanine aminotransferase (ALT) \\
\hline Total and direct bilirubin \\
\hline Alkaline phosphatase (ALP) \\
\hline Gamma-glutamyl-transferase (GGTP) \\
\hline Albumin or total protein \\
\hline Cholesterol \\
\hline Glucose
\end{tabular}

reason, all patients with cystic fibrosis should be observed carefully for the occurrence of this complication in the first decade of life. This is especially applicable for patients with a high risk of CFLD development, i.e. those of male gender, with a history of meconium ileus and the presence of severe CFTR gene mutations [8]. Table II provides a list of laboratory tests for CFLD occurrence, which should be performed annually in patients with cystic fibrosis. In terms of the symptoms and complications of the respiratory system, the diagnosis of CFLD does not significantly change the clinical course of the disease. However, in some patients a rapid clinical deterioration may occur. The nutritional status of the patient should be also assessed systematically [8].

\section{Treatment of cystic fibrosis-associated liver disease}

The CFLD treatment should be comprehensive and carried out by a multi-specialty team (gastroenterologist, hepatologist, dietitian, radiologist and surgeon). Its main aim should be to prevent liver damage and complications related to portal hypertension and cirrhosis. There is no CFLD treatment of proven long-term efficacy. Ursode- oxycholic acid (UDCA) is recommended for any patient with cystic fibrosis, and a pathology of the hepatobiliary system, but there is no strong evidence of its efficacy in preventing cirrhosis of the liver. There are four suggested UDCA mechanisms of action: (1) it displaces toxic bile acids from enterohepatic circulation, (2) it has a stabilising/cytoprotective effect on cell membranes exposed to toxic bile acids, (3) it stimulates the Ca-activated chloride channels, and (4) it has immunoregulatory properties. It is believed that UDCA has a beneficial effect on the activity of liver enzymes, the secretory function of the liver, biliary drainage, the "histology" of the liver and the profile of essential fatty acids. Moreover, UDCA shows choleretic and cholagogic activity, reduces the lithogenicity of bile and prevents bacterial translocation and the development of endotoxaemia. Studies have shown that the most efficient dose of UDCA is $20-30 \mathrm{mg} / \mathrm{kg}$ body weight/day - higher than the doses used in other liver diseases. It has also been shown that long-term treatment with UDCA can delay the progression of cirrhotic changes and even reverse existing changes of focal biliary cirrhosis type $[1,14]$.

In all patients with cystic fibrosis, including those in whom CFLD has developed, a well balanced diet including fat-soluble vitamin supplementation is necessary. The supply of protein should not be routinely restricted, unless there are signs of decompensated liver failure and hepatic encephalopathy.

\section{Liver transplantation}

In patients with an advanced stage of CFLD (decompensated liver failure), transplantation is a treatment of choice. In patients with advanced bronchopulmonary changes in the course of CF, multiple-organ transplant is routinely performed: the heart, the lungs and the liver. This procedure allows one to maintain or to improve the lung function, as well as having a beneficial effect on the nutritional status of the patient. A significant limitation of this procedure relates to the side effects of using immunosuppressive drugs, such as disorders of

Table III. Theoretical approaches to therapy for CFLD [13]

\begin{tabular}{ll} 
Aim & Approach \\
\hline Replacement of the defective gene & Gene transfer mediated by adenovirus or liposomes \\
\hline Stimulation of accessory Cl pathways & Agonists of Cl channels (purinogenic nucleotides, UDCA?) \\
\hline Reduction of inflammatory response & Anti-inflammatory agents \\
\hline Reduction of fibrogenesis & $\begin{array}{l}\text { Colchicine, antioxidants, steroids, interferon, growth factor } \\
\text { modulators }\end{array}$ \\
\hline Reduction of hepatocellular damage & $\begin{array}{l}\text { Antioxidants, UDCA, glutathione, avoiding malnutrition, } \\
\text { antiviral prophylaxis }\end{array}$ \\
\hline Stimulation of bile flow at the level of hepatocyte & UDCA, glutathione
\end{tabular}


hematopoietic system functions, abnormalities of the lipid and carbohydrate metabolism or nephrotoxicity [2].

Recently, researchers have some hope in one of the new therapeutic options, ATALUREN (PTC124), which affects the translation processes in ribosomes, inducing the production of a "working" CFTR protein [15] However, causal treatment of cystic fibrosis and its complications (including CFLD) is not possible although researchers are still looking for new therapeutic methods. Some of these therapeutic options, which may be applied in the treatment of CFLD in the near future, are presented in Table III.

The world of science is currently focused on the early clinical, biochemical and radiological markers of CFLD, as well as implementing new therapeutic options resulting from the better understanding of the pathophysiology of liver damage and fibrosis processes in the course of CFLD. Multicentre, randomised, prospective studies are also needed to assess the efficacy of UDCA use in the prevention of CFLD. Further progress is necessary in the field of liver transplantation, with the evaluation of distant results considering the life quality of the recipient.

\section{References}

1. Siano M, De Gregorio F, Boggia B, et al. Ursodeoxycholic acid treatment in patients with cystic fibrosis at risk for liver disease. Dig Liver Dis 2010; 42: 428-31.

2. Diwakar V, Pearson L, Beath S. Liver disease in children with cystic fibrosis. Paediatr Respir Rev 2001; 2: 340-9.

3. Herrmann U, Dockter G, Lammert F. Cystic fibrosis-associated liver disease. Best Pract Res Clin Gastroenterol 2010; 24: 585-92.

4. Minagawa N, Nagata J, Shibao K, et al. Cyclic AMP regulates bicarbonate secretion in cholangiocytes through release of ATP into bile. Gastroenterology 2007; 133: 1592-602.

5. Fiorotto R, Spirlì C, Fabris L, et al. Ursodeoxycholic acid stimulates cholangiocyte fluid secretion in mice via CFTR-dependent ATP secretion. Gastroenterology 2007; 133: 1603-13.

6. Majka L, Pogorzelski A, Mtynarczyk W, et al. Effect of genotype on selected clinical features of Polish cystic fibrosis adults. J Appl Genet 2001; 42: 367-77.

7. McKone EF, Emerson SS, Edwards KL, et al. Effect of genotype on phenotype and mortality in cystic fibrosis: a retrospective cohort study. Lancet 2003; 361: 1671-6.

8. Colombo C, Battezzati PM, Crosignani A, et al. Liver disease in cystic fibrosis: a prospective study on incidence, risk factors and outcome. Hepatology 2002; 36: 1374-82.

9. Lindblad A, Glaumann H, Strandvik B. Natural history of liver disease in cystic fibrosis. Hepatology 1999; 30: 1151-8.

10. Shapira R, Hadzic N, Francavilla R, et al. Retrospective review of cystic fibrosis presenting as infantile liver disease. Arch Dis Child 1999; 81: 125-8.

11. Corbett K, Kelleher S, Rowland M, et al. Cystic fibrosis-associated liver disease: a population-based study. J Pediatr 2004; 145: 327-32.
12. Colombo C, Crosignani A, Battezzati PM. Liver involvement in cystic fibrosis. J Hepatol 1999; 31: 946-54.

13. Littlewood JM. Good care for people with cystic fibrosis. Paediatr Respir Rev 2000; 1: 179-89.

14. Lamireau T, Monnereau S, Martin S, et al. Epidemiology of liver disease in cystic fibrosis: a longitudinal study. J Hepatol 2004; 41: 920-5.

15. Wilschanski M, Miller LL, Shoseyov D, et al. Chronic ataluren (PTC124) treatment of nonsense mutation cystic fibrosis. Eur Respir J 2011; 38: 59-69.

Received: 26.10 .2011

Accepted: 25.11 .2012 